THERAPEUTICS

\title{
Susceptibility or resistance?
}

Somatic mutations in the epidermal growth factor receptor (EGFR) are often detected in non-small-cell lung tumours, and these can determine a patient's response to drugs that target this receptor tyrosine kinase, such as gefitinib (Iressa) and erlotinib (Tarceva). In a search for other genetic factors that might determine how patients respond to these drugs, William Pao et al. discovered that a large percentage of patients with lung adenocarcinomas that were resistant to gefitinib or erlotinib harboured mutations in KRAS.

KRAS is a downstream factor of many receptor tyrosine kinases, including EGFR. Like EGFR, KRAS is mutated in many lung cancers (15-30\%), but very few tumours carry mutations in both genes, indicating that they have functionally equivalent roles in tumorigenesis. As certain EGFR mutations have been shown to make lung tumours more sensitive to gefitinib and erlotinib, Pao et al. searched for other mutations in this signalling pathway that might affect patient response.

They categorized 60 lung adenocarcinoma samples from patients as sensitive or refractory to single-agent gefitinib or erlotinib therapy. These were then evaluated for mutations in KRAS, as well as EGFR as a control. Of the 22 samples taken from patients whose tumours were sensitive to either drug, none had KRAS mutations. When they looked at tumour samples from patients who were refractory to either therapy, however, 9 of 38 (24\%) carried a KRAS mutation. Mutations in exon 2 of
$K R A S$ were correlated with resistance to either drug, although the incidence of KRAS mutations in patients treated with erlotinib was low (only 4 tumours). Consistent with previous studies, however, $77 \%$ of drugsensitive tumours had EGFR mutations, whereas none of the tumour samples taken from patients who were refractory to treatment carried mutations in this gene.

These findings mean that patients with lung adenocarcinomas that carry KRAS mutations are not likely to experience tumour regression if treated with either drug. The findings will have to be validated, however, in a large prospective trial. Further studies are also required to determine how KRAS mutations mediate resistance to EGFR inhibitors.

Kristine Novak

60) References and links ORIGINAL RESEARCH PAPER Pao, W. et al. KRAS mutations and primary resistance of lung adenocarcinomas to gefitinib or erlotinib. PLoS Med. 2, 57-61 (2005) FURTHER READING Herbst, R. S., Fukuoka, M. \& Baselga, J. Gefitinib - a novel targeted approach to treating cancer. Nature Rev. Cancer 4, 956-965 (2004)

\section{METABOLISM}

\section{Clash of therapies?}

The response of women with breast cancer to adjuvant treatment with tamoxifen varies widely, and a common side effect of tamoxifen is hot flushes. David Flockhart and colleagues have now found that interactions between tamoxifen-metabolizing enzymes and drugs given to treat hot flushes might affect tamoxifen activity.

A total of 80 patients with newly diagnosed breast cancer who were starting tamoxifen therapy were genotyped for common functional polymorphisms in the cytochrome P450 (CYP) enzymes CYP3A5, CYP2D6 and CYP2C9. In vitro data show that the metabolism of tamoxifen to its primary metabolite $\mathrm{N}$-desmethyltamoxifen occurs through CYP3A5, whereas the metabolism of tamoxifen to the active metabolites endoxifen and 4-hydroxytamoxifen occurs mainly through CYP2D6. Twenty-four of the patients were also taking selective serotonin re-uptake inhibitors, which inhibit the CYP2D6 isoform, for the treatment of hot flushes.

Measurements at 1 and 4 months after the start of tamoxifen treatment showed that the wild-type or a variant form of CYP2C9 or CYP3A5 did not affect plasma concentrations of the different metabolites. By contrast, there was an association with CYP2D6 genotype.
Analysis of CYP2D6 genotypes revealed that 48 patients were homozygous for the wildtype allele $\left(C Y P 2 D 6^{\star} 1\right.$, referred to as wt/wt). Twenty-nine patients had wt/variant (vt) genotypes and three patients had vt/vt genotypes, all of which result in non-functional proteins. The CYP2D6 genotype was associated with changes in active endoxifen concentrations but not with concentrations of tamoxifen or the other metabolites. Patients with wt/vt genotypes had endoxifen concentrations that were reduced to $55 \%$ of levels found in patients with the wt/wt genotype. The vt/vt genotype had concentrations only $26 \%$ of those measured in patients with the wt/wt genotype.

So, what difference did concomitant use of CYP2D6 inhibitors make? Of the 24 women taking CYP2D6 inhibitors, those with the wt/wt genotype had concentrations of endoxifen 58\% lower than wt/wt patients not taking these drugs. The wt/vt variant group taking CYP2D6 inhibitors had mean plasma endoxifen concentrations 38\% lower than wt/vt variants not taking these drugs. When the types of drug were further analysed it was found that of those patients $(30 \%)$ with wt/wt variants taking selective serotonin re-uptake inhibitors, taking paroxetine in particular substantially lowered endoxifen concentrations.

Co-administration of drugs that inhibit CYP2D6 could therefore affect the anticancer effectiveness of tamoxifen, and this needs further testing linked to clinical outcome in clinical trials.

$$
\text { Ezzie Hutchinson }
$$

\section{(4) References and links}

ORIGINAL RESEARCH PAPER Minn, A. J. et al. Distinct organ-specific metastatic potential of individual breast cancer cells and primary tumors. J. Clin. Invest. 115, 44-55 (2005) WEB SITE

David Flockhart's lab: http://medicine.iupui.edu/clinical/

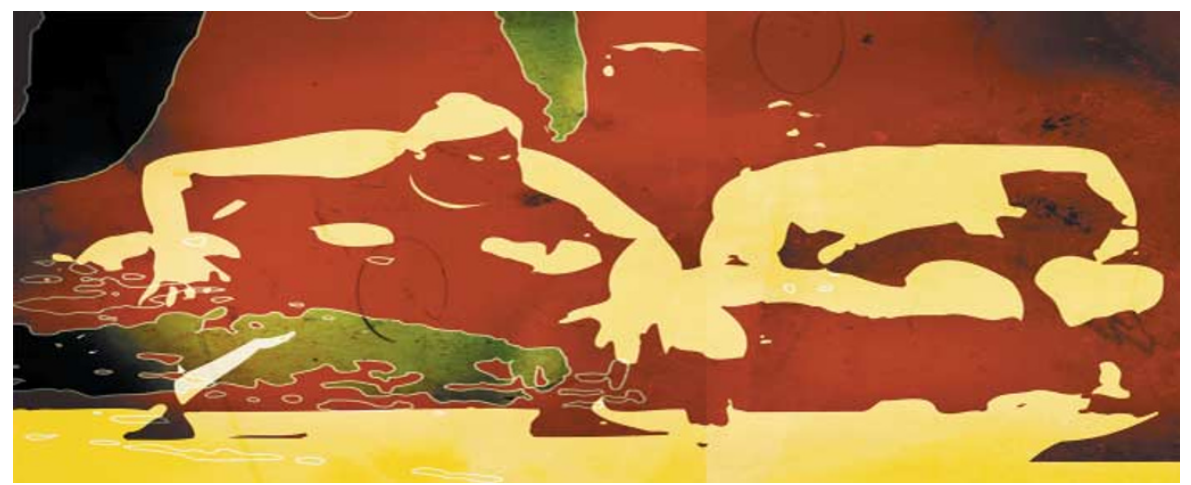

